\title{
Apolipoprotein D in CD34-positive and CD34-negative cutaneous neoplasms: a useful marker in differentiating superficial acral fibromyxoma from dermatofibrosarcoma protuberans
}

\author{
Mikhail Lisovsky ${ }^{1}$, Mai P Hoang ${ }^{1}$, Karen A Dresser ${ }^{1}$, Payal Kapur², Jag Bhawan $^{3}$ \\ and Meera Mahalingam ${ }^{1}$
}

${ }^{1}$ Department of Pathology, UMass Medical School, Worcester, MA, USA; ${ }^{2}$ University of Texas Southwestern Medical Center, Dallas, TX, USA and ${ }^{3}$ Department of Dermatology, Boston University School of Medicine, Boston, MA, USA

\begin{abstract}
More recent techniques to characterize the genetic profile of soft-tissue tumors include the use of gene arrays. Using this technique, Apolipoprotein D (Apo D), a 33-kDa glycoprotein component of high-density lipoprotein, has been found to be highly expressed in dermatofibrosarcoma protuberans. To corroborate these results, we sought to ascertain the utility of Apo D by investigating its sensitivity and specificity in a variety of CD34positive and CD34-negative cutaneous neoplasms including superficial acral fibromyxoma, sclerotic fibromas, and cellular dermatofibromas. Of interest, we found absence of Apo D expression in all four cases of superficial acral fibromyxoma. Of the remaining CD34-positive lesions, Apo D expression was noted in 35/36 (97\%) cases of dermatofibrosarcoma protuberans, $3 / 5(60 \%)$ giant-cell fibroblastomas, $4 / 4(100 \%)$ sclerotic fibromas, $8 / 8$ (100\%) neurofibromas, and $1 / 1(100 \%)$ solitary fibrous tumor. Of the CD34-negative lesions, Apo D expression was noted in 2/22 (9\%) regular dermatofibroma, 23/45 (51\%) cellular dermatofibroma, $10 / 10(100 \%)$ malignant fibrous histiocytoma, $9 / 10(90 \%)$ atypical fibroxanthoma, $7 / 8$ (86\%) cellular neurothekeoma, 9/9 (100\%) malignant melanoma, $8 / 8(100 \%)$ melanocytic nevi (100\%), 0/2 superficial angiomyxoma, 0/15 fibromatosis, 0/ 1 nodular fasciitis, and $1 / 2(50 \%)$ desmoplastic fibroblastomas. In summary, our findings indicate that Apo D expression is not specific to dermatofibrosarcoma protuberans. Its principal use as an immunohistochemical adjunct lies in its utility in differentiating superficial acral fibromyxoma from dermatofibrosarcoma protuberans. Although strong positive staining of Apo D in a markedly atypical fibrohistiocytic lesion is suggestive of atypical fibroxanthoma and/or malignant fibrous histiocytoma, further studies with the inclusion of other atypical spindled cell neoplasms are required to conclusively prove the same.
\end{abstract}

Modern Pathology (2008) 21, 31-38; doi:10.1038/modpathol.3800971; published online 21 September 2007

Keywords: apolipoprotein D; Apo D; CD34; superficial acral fibromyxoma; dermatofibrosarcoma protuberans

Apolipoprotein D (Apo D), a protein component of the human plasma lipid transport system, was first isolated and characterized by McConathy and Alaupovic. $^{1}$ It is a $30-\mathrm{kDa}$ glycoprotein that is

Correspondence: Dr M Mahalingam, MD, PhD, FRCPath, Dermatopathology Section, Department of Dermatology, Skin Pathology Laboratory, Boston University School of Medicine, 609 Albany Street, J-308, Boston, MA 02118, USA.

E-mail: mahalinm@bu.edu

Presented in part at the 96th United States and Canadian Academy of Pathology Annual Meeting, San Diego, CA, USA, March 2007.

Received 31 May 2007; revised 18 July 2007; accepted 1 August 2007; published online 21 September 2007 mainly associated with high-density lipoproteins and forms disulfide-linked heterodimers with other lipoproteins. ${ }^{2}$ While the functional role of Apo D in the metabolism of plasma lipoproteins remains unclear, its ability to form complexes with lecithin:cholesterol acyltransferase suggests that it may be involved in cholesterol esterification and transport of substrates. ${ }^{3,4}$ Apo D mRNA has been detected in a number of different tissues including liver, intestine, pancreas, kidney, placenta, adrenal, spleen, and fetal brain. ${ }^{5}$ More recent techniques to study soft tissue tumors include the use of DNA microarray analysis. ${ }^{6}$ Using this technique, Linn et $a l^{6}$ found Apo D to be not only highly expressed 
in dermatofibrosarcoma protuberans (9/9 cases, $100 \%$ ) but also that expression of Apo D in dermatofibrosarcoma protuberans appeared to be relatively specific. ${ }^{7}$

While the histologic features of dermatofibrosarcoma protuberans are fairly distinct, that is a storiform or cartwheel proliferation of spindled neoplastic cells extending into the subcutis, the same can be difficult to appreciate in a superficial biopsy, and often requires resorting to the use of adjunct immunohistochemical tools. ${ }^{8,9}$ Expression of CD34, the human progenitor cell antigen and the most commonly used ancillary stain to confirm a histologic diagnosis of dermatofibrosarcoma protuberans, is reportedly positive in most cases of dermatofibrosarcoma protuberans with a sensitivity ranging from 66.6 to $100 \% .{ }^{10,11}$ Literature indicates that up to $30 \%$ of cases of dermatofibrosarcoma protuberans may be CD34 negative. ${ }^{10,11}$ This high value may be a reflection of inclusion of cases of cellular dermatofibroma misdiagnosed as dermatofibrosarcoma protuberans; and if one includes cases of dermatofibrosarcoma protuberans that are focally positive for CD34, this number is more likely to be well under $10 \%$. Further confounding this issue is the fact that expression of CD34 is not entirely specific to dermatofibrosarcoma protuberans and may be found in a variety of other tumors including superficial acral fibromyxoma and sclerotic fibromas-all potential histologic mimics of dermatofibrosarcoma protuberans. ${ }^{5}$ The biologic course of all of these 'non-dermatofibrosarcoma protuberans
CD34-positive' lesions is however, indolent and thus fairly different from that of dermatofibrosarcoma protuberans, a locally aggressive tumor requiring wide surgical excision. While metastases is relatively uncommon in dermatofibrosarcoma protuberans, it has been noted, albeit rarely, in cases with a history of multiple recurrences. ${ }^{12}$

In this study we sought to ascertain the utility of Apo D in differentiating dermatofibrosarcoma protuberans from its CD34-positive histologic mimics. We also investigated the sensitivity and specificity of Apo D by ascertaining its expression in a variety of other CD34-positive and CD34-negative cutaneous neoplasms including cellular dermatofibromas.

\section{Materials and methods}

The study was approved by the UMass Medical Center institutional review board (IRB \# H-12235). Archival materials from 185 cases (Table 1) were retrieved from the pathology files of UMass Medical Center, Worcester, MA. Five cases of giant cell fibroblastoma were retrieved from the pathology files of University of Texas Southwestern Medical Center, Dallas, TX, USA. The histologic sections of all cases were re-reviewed and the diagnoses confirmed by two dermatopathologists (MM and $\mathrm{MH})$. All patient data were de-identified.

Five-micrometer-thick sections were cut for immunohistochemical studies, which were performed

Table 1 Immunohistochemical results

\begin{tabular}{|c|c|c|c|c|c|c|c|}
\hline \multirow[t]{2}{*}{ Diagnosis } & \multirow[t]{2}{*}{ Number of cases } & \multicolumn{3}{|c|}{ Apo D } & \multicolumn{3}{|c|}{$C D 34$} \\
\hline & & $3+$ & $2+$ & Neg & $3+$ & $2+$ & Neg \\
\hline \multicolumn{8}{|l|}{ CD34-positive lesions } \\
\hline Dermatofibrosarcoma protuberans & 36 & 30 & 5 & 1 & 33 & 3 & \\
\hline Giant cell fibroblastoma & 5 & 1 & 2 & 2 & & 4 & 1 \\
\hline Superficial acral fibromyxoma & 4 & & & 4 & 4 & & \\
\hline Sclerotic fibroma & 4 & 3 & 1 & & 4 & & \\
\hline Neurofibroma & 8 & 8 & & & 5 & 1 & 2 \\
\hline Solitary fibrous tumor & 1 & 1 & & & 1 & & \\
\hline \multicolumn{8}{|l|}{ Dermatofibroma } \\
\hline Regular dermatofibroma & 22 & & 2 & 20 & & 1 & 21 \\
\hline Cellular dermatofibroma & 45 & 11 & 12 & 22 & & 5 & 40 \\
\hline \multicolumn{8}{|l|}{ Atypical fibrohistiocytic proliferations } \\
\hline Malignant fibrous histiocytoma & 10 & 9 & 1 & & & 1 & 9 \\
\hline Atypical fibroxanthoma & 10 & 8 & 1 & 1 & & & 10 \\
\hline \multicolumn{8}{|l|}{ Other lesions } \\
\hline Cellular neurothekeoma & 8 & 2 & 5 & 1 & & & 8 \\
\hline Superficial angiomyxoma & 2 & & & 2 & & & 2 \\
\hline Superficial fibromatosis & 15 & & & 15 & & & 15 \\
\hline Nodular fasciitis & 1 & & & 1 & & & 1 \\
\hline Desmoplastic fibroblastoma & 2 & & 1 & 1 & & & 2 \\
\hline Malignant melanoma & 9 & 7 & 2 & & 1 & & 8 \\
\hline Melanocytic nevus & 8 & 6 & 2 & & 1 & 1 & 6 \\
\hline
\end{tabular}


using the standard techniques, heat-induced epitope retrieval buffer, and primary antibodies against Apo D (36C6, 1:40 dilution, Novocastra, Newcastle-on-Tyne, UK) and CD34 (QBend-10, 1:160 dilution, Dako Cytomation, Carpinteria, CA, USA). Appropriate positive and negative controls were included.

Positive staining, noted by ascertaining expression of ApoD in the cytoplasm, was scored as $3+$ (greater than $50 \%$ of the tumor cells), $2+(10-49 \%$ ) or $1+/$ negative (less than $10 \%$ ). Significant nuclear staining was not noted. The slides were reviewed by two dermatopathologists (MPH, MM), and disagreements were reviewed together to achieve a consensus score.

\section{Results}

\section{CD34-Positive Neoplasms}

\section{Dermatofibrosarcoma protuberans}

Microscopic examination of all of the cases of dermatofibrosarcoma protuberans (36) showed a superficial and deep dermal ill-circumscribed proliferation of spindled cells arranged in a storiform pattern with extension into the subcutaneous tissue (Figure 1a). While cytologic atypia and mitotic figures were not apparent in any of the cases, prominent myxoid change (Figure 1d) was noted in 2 of the 36 cases of which one occurred on acral skin (Figure 2a and b). Thirty-five of thirty-six cases (97\%) were positive for Apo D (3+, 30 cases; $2+, 5$ cases) (Figure $1 \mathrm{~b}$ and e). All cases were positive for CD34 (100\%) (Figure 1c and f).

\section{Superficial acral fibromyxoma}

Histologic features of all of the cases of superficial acral fibromyxoma (4) are typified by the case shown (Figures 1g and 2c). Cytologic atypia and mitotic figures were not apparent in any of the cases (Figure 2d). All four cases were negative for Apo D (Figure 1h) and positive for CD34 (Figure 1i).

\section{Giant-cell fibroblastoma}

Histologic features of all of the cases of giant-cell fibroblastomas (5) are typified by the case shown (Figure 1j). Cytologic atypia and mitotic figures were not apparent in any of the cases. Apo D (Figure 1k) was positive in three of five cases $(3+, 1$ case; $2+, 2$ cases). Of the two ApoD negative cases, one was also negative for CD34. Four cases were positive for CD34 (Figure 1l).

\section{Others}

Positive staining with ApoD was also noted in sclerotic fibroma (4/4), neurofibroma (8/8) and in the only case of solitary fibrous tumor (1/1). Overall, staining with CD34 paralleled that of ApoD with the exception of neurofibroma in which two cases were negative for CD34.

\section{CD34-Negative Neoplasms}

\section{Dermatofibroma}

This category included cellular dermatofibroma (45) (which included eight cases of the aneurysmal variant) and regular dermatofibroma (22) (of which deep extension into the subcutaneous tissue in a pushing but not 'honeycombed' pattern was noted in 5) (Figure 3a). Cytologic atypia was minimal in all cases of cellular dermatofibroma and, rare, albeit normal, mitotic figures (up to $3 / 10 \mathrm{HPFs}$ ) were noted in a few of the cases. Of the 45 cases of cellular dermatofibroma, Apo D positivity was $3+$ in 11 cases and $2+$ in 12 cases (Figure $3 \mathrm{~b}$ ). Two of 22 regular dermatofibroma showed $(2+)$ Apo D positivity. CD34 was focally positive in five cellular dermatofibroma cases (Figure 3c) and 1 regular dermatofibroma.

Atypical fibro-histiocytic neoplasms

Histologic features of all of the cases of atypical fibroxanthoma (10) (Figure 3d) and malignant fibrous histiocytoma (10) (Figure 3g) are shown. Positive staining with Apo D expression was noted in $9 / 10$ atypical fibroxanthoma $(3+, 8$ cases; $2+, 1$ case) (Figure 3e) and in 10/10 malignant fibrous histiocytoma ( $3+, 9$ cases; $2+, 1$ case) (Figure $3 \mathrm{~h}$ ). One malignant fibrous histiocytoma case focally expressed CD34. The remaining nine malignant fibrous histiocytoma cases and all 10 atypical fibroxanthoma cases were negative for CD34 (Figure $3 f$ and i).

\section{Nevomelanocytic proliferation}

This category included malignant melanoma (9) (Figure 3j) and melanocytic nevi (8). The latter included Spitz nevi (4), congenital nevus (1), compound nevus (1), and intradermal nevi (2). Apo D expression was noted in all nine cases of malignant melanoma ( $3+, 7$ cases; $2+, 2$ case) (Figure 3k) and all eight cases of melanocytic nevus ( $3+, 6$ cases; $2+, 2$ cases). CD34 was positive in 1 case of malignant melanoma (Figure 3l) and in two cases of melanocytic nevus.

\section{Others}

Positive staining with Apo D was also noted in cellular neurothekeoma (7/8), superficial angiomyxoma (2/2), nodular fasciitis (1/1), superficial fibromatosis (15/15), and desmoplastic fibroblastomas (1/2). All cases were negative for CD34.

\section{Discussion}

A histologic giveaway to the diagnosis of dermatofibrosarcoma protuberans, a spindled-cell, fasciculated neoplasm typically extending into the subcutis is the 'cartwheel' appearance-referring to the central acellular focus with radiating fascicles of cells, best appreciated at low magnification. ${ }^{13}$ Typically lesions of dermatofibrosarcoma protuber- 


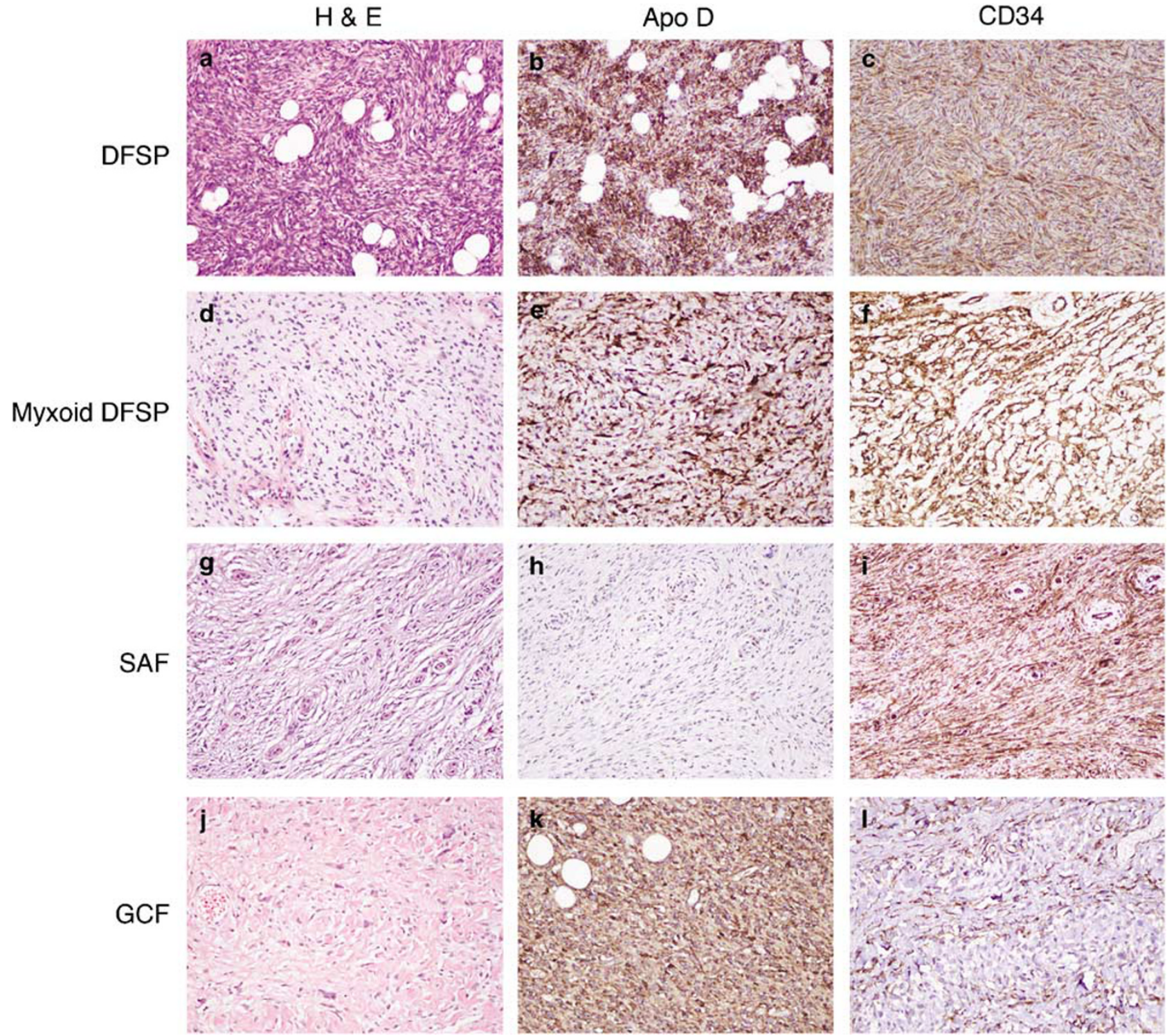

Figure 1 CD34-positive neoplasms: H\&E (hematoxylin-eosin) $(\times 20)$, Apo D $(\times 20)$ and CD34 $(\times 20)$. DFSP (dermatofibrosarcoma protuberans), Myxoid DFSP, SAF (superficial acral fibromyxoma), and GCF (giant-cell fibroblastoma).

ans are bland cytologically and it is the architecture (deep infiltrate and cartwheeling) and presence of predominantly of spindled cells that are diagnostic histopathologic clues. However, an appreciation of the former can be difficult, if not impossible, in a superficial shave biopsy and more often than not necessitates the use of ancillary tools such as immunohistochemistry. CD34-reactivity in dermatofibrosarcoma protuberans was first reported in 1990 by Ramani et al. ${ }^{14}$ This human progenitor antigen is the most widely used immunohistochemical stain due to its 'supposedly' consistent expression in dermatofibrosarcoma protuberans. ${ }^{15}$ While initial studies found CD34 positivity in all cases of dermatofibrosarcoma protuberans, subsequent reports indicate that a proportion may be negative for this marker. ${ }^{10,11,15}$ Confounding the issue further is the fact that expression of CD34 is not unique to dermatofibrosarcoma protuberans and may be seen in a wide variety of lineage un-related, benign, and malignant neoplasms. ${ }^{15}$ More recently, gene microarrays have been used to determine mRNA expression in various tumors. ${ }^{6}$ Using this technique, Linn et $a l^{6}$ found Apo D to be highly expressed in all nine cases of CD34 positive dermatofibrosarcoma protuberans indicating that its utility paralleled that of CD34 as an immunohistochemical adjunct in the workup of dermatofibrosarcoma protuberans. Findings from our current study corroborate those of Linn et $a l^{6}$ in that we found Apo D to be indeed highly expressed in dermatofibrosarcoma protuberans $(97 \%)$.

In addition to dermatofibrosarcoma protuberans, we found Apo D expression in other benign and malignant including locally aggressive neoplasms (see Table 1). Thus, our own experience, not entirely 

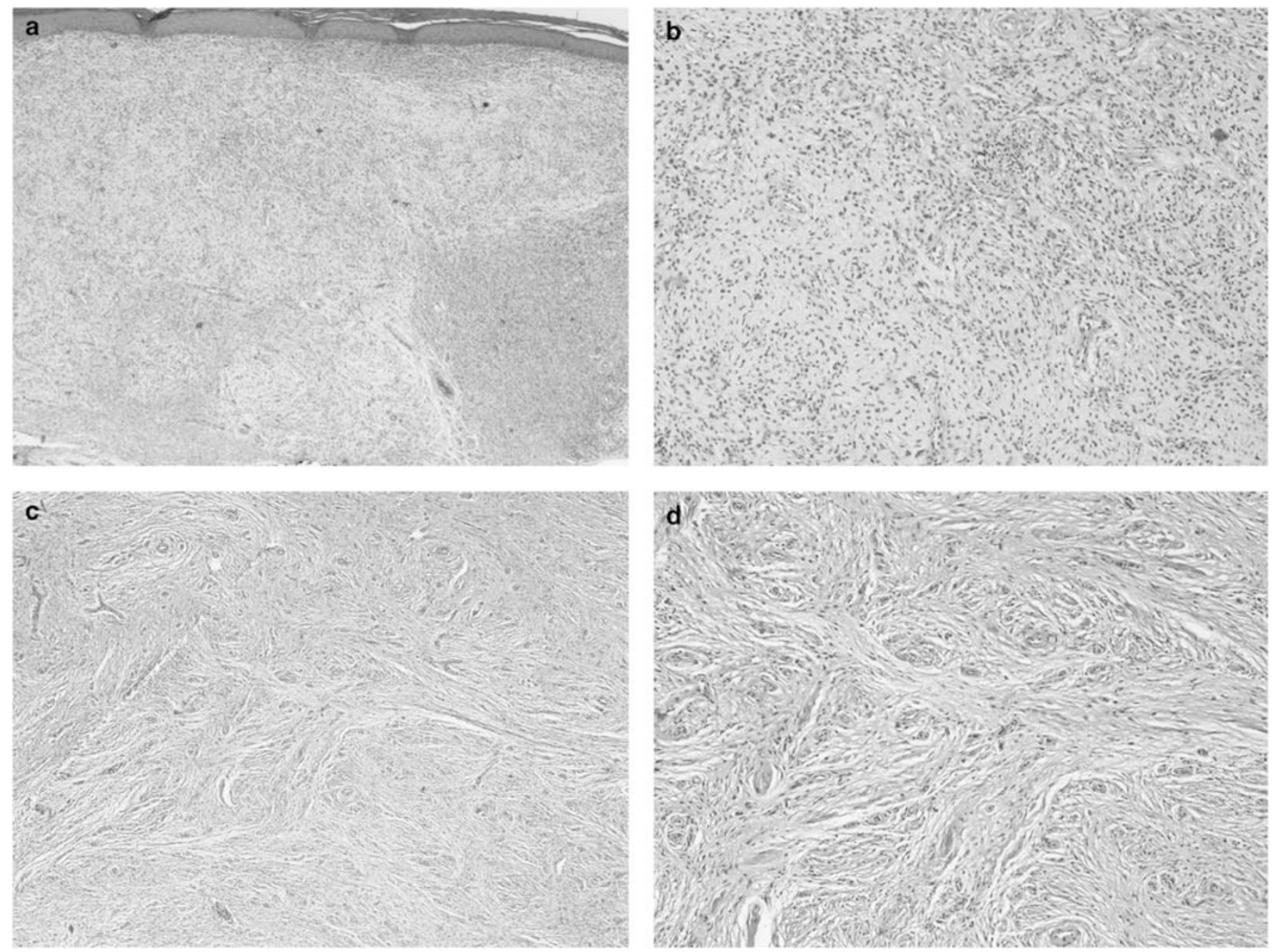

Figure 2 Histologic features of myxoid dermatofibrosarcoma protuberans at $\times 4$ (a) and $\times 10$ (b), and superficial acral fibromyxoma at $\times 4(\mathbf{c})$ and $\times 10(\mathbf{d})$.

unlike that of West et $a l^{7}$ is that expression of Apo D is not entirely specific for dermatofibrosarcoma protuberans. They also noted strong Apo D immunoreactivity in non-dermatofibrosarcoma protuberans entity cases including some cases of alveolar soft part sarcoma, glomus tumor, granular cell tumor, endometrial stromal sarcoma, angiosarcoma, neurofibroma, tenosynovial giant cell tumor, and atypical lipomatous tumors. ${ }^{7}$ However, most of these entities are fairly distinct from a histopathologic perspective and not routinely encountered in the skin. The only one that can perhaps be misdiagnosed as dermatofibrosarcoma protuberans, especially if the biopsy is a superficial one is sclerotic fibroma. ${ }^{16}$ All four sclerotic fibromas in our study were Apo D positive; thus speaking against the utility of Apo $D$ in differentiating sclerotic fibromas from dermatofibrosarcoma protuberans. Oddly enough, only in the case of cellular dermatofibroma with a central wellcircumscribed sclerotic fibroma-like area, Apo D was diffusely and strongly positive throughout the lesion but not in the sclerotic fibroma-like area (see Figure $3 b)$.
Our experience of Apo D expression in 60\% of giant cell fibroblastomas, a rare pediatric tumor and one believed to be a variant of dermatofibrosarcoma protuberans, ${ }^{17,18}$ is similar to that $(67 \%)$ by West et al. ${ }^{7}$

Perhaps, the most significant finding in our study was the absence of Apo D expression in all of the cases of superficial acral fibromyxoma. Superficial acral fibromyxoma is a relatively uncommon, distinctive entity with a predilection for the fingers and toes of adults. ${ }^{19}$ Unlike dermatofibrosarcoma protuberans, superficial acral fibromyxoma follows a benign biologic course. ${ }^{19}$ In Fetchs' original study, of the 21 (of 37) cases that had follow-up, only one recurred, two persisted or progressed after local excision, and none metastasized. ${ }^{19}$ Dermatofibrosarcoma protuberans on the other hand is locally aggressive with a very real potential for local recurrence if incompletely excised. ${ }^{13}$ Differentiating superficial acral fibromyxoma from dermatofibrosarcoma protuberans, especially its myxoid variant, is not a diagnostic dilemma if the biopsy is deep where the storiform pattern and infiltrative architecture pathognomonic of dermatofibrosarcoma 

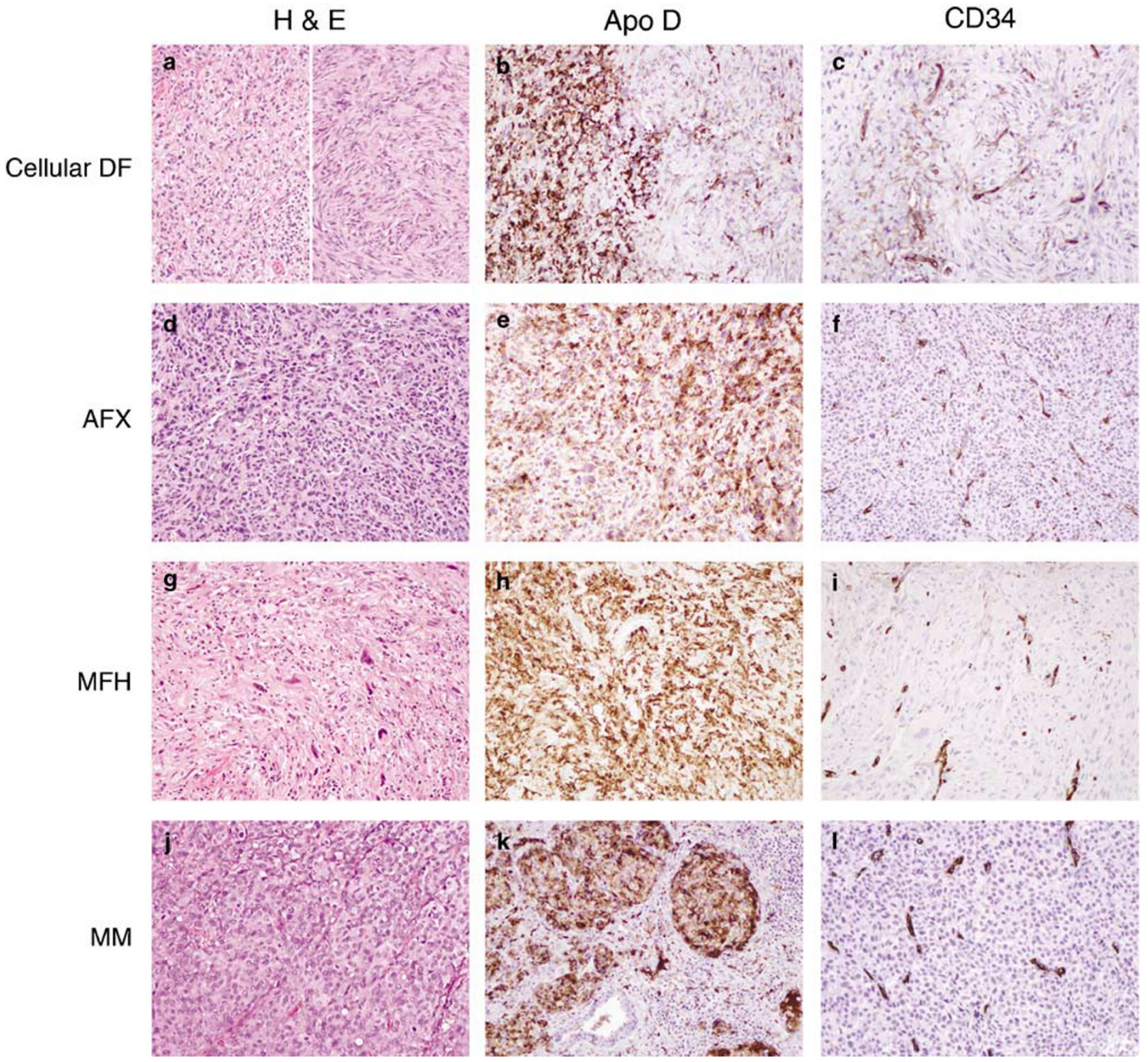

Figure 3 CD34-negative neoplasms: H\&E (hematoxylin-eosin) $(\times 20)$, Apo D $(\times 20)$ and CD34 $(\times 20)$. Cellular DF (dermatofibroma), AFX (atypical fibroxanthoma), MFH (malignant fibrous histiocytoma), and MM (malignant melanoma).

protuberans are recognized. However, the distinction can be difficult, if not impossible in a superficial biopsy, given the following; both may occur at the same site (dermatofibrosarcoma protuberans has, albeit rarely, been reported on acral skin in approximately $3 \%$ of cases). ${ }^{12,19}$ Both can have a myxoid histologic appearance and more importantly both express CD34. One of the cases of superficial acral fibromyxoma in the current study was indeed misdiagnosed as a myxoid dermatofibrosarcoma protuberans based on the stroma and CD34 positivity (Figure 2c and d). Although the differential diagnosis of a mildly cellular neoplasm in a myxoid stroma also includes neurofibroma (also CD34 and ApoD positive) and superficial angiomyxoma (CD34 and Apo D negative), these are not as much of a histological dilemma.

Cellular dermatofibromas that extend into the subcutis need to be definitively differentiated from dermatofibrosarcoma protuberans. This distinction is possible with the use of the immunohistochemical marker CD34. ${ }^{20,21}$ However, CD34 has been reported to be expressed, albeit focally, up to $40 \%$ cases of benign fibrous histiocytoma. ${ }^{21,22}$ We found that up to $50 \%$ of cellular dermatofibroma contained a subpopulation of ApoD-positive cells . Negative staining with CD34 was observed in $37 \%$ of these cases. Thus, Apo D is of no real utility in differentiating dermatofibrosarcoma protuberans from cellular dermatofibroma as it lacks specificity. 
Furthermore, although the utility of CD34 appears limited because of its lack of specificity, from a practical perspective it still is a more helpful stain in differentiating cellular dermatofibroma from dermatofibrosarcoma protuberans.

Our own experience with expression of Apo D in malignant fibrous histiocytoma is in contrast to that by West et al. ${ }^{7}$ They found only $38 \%$ of their malignant fibrous histiocytoma cases to be positive for Apo D with weak expression in $95 \%$ of the positive cases. ${ }^{7}$ All of our cases of malignant fibrous histiocytoma and atypical fibroxanthoma were diffusely and strongly positive for Apo D lending credence to the concept that perhaps these are not two distinct entities but a spectrum of the same. ${ }^{13}$ For now, both malignant fibrous histiocytoma and atypical fibroxanthoma remain diagnoses of exclusion. Our own experience with recently proposed markers of utility for the same such as CD10 and CD99 is that they are inconsistently expressed and also expressed in a number of other lineageunrelated neoplasms. ${ }^{23-26}$ Speculative as it may seem, given that we did not study ApoD expression in other atypical spindled cell neoplasms, the consistently strong positive Apo D staining $(3+$, $80 \%$ ) in cases of atypical fibroxanthoma suggests that this marker holds some promise in terms of diagnostic utility.

In summary, our findings indicate that expression of Apo D is not specific to dermatofibrosarcoma protuberans. For now, its principal use as an immunohistochemical adjunct is that it is of utility in differentiating superficial acral fibromyxoma from dermatofibrosarcoma protuberans.

\section{References}

1 McConathy WJ, Alaupovic P. Isolation and partial characterization of apolipoprotein D: a new protein moiety of the human plasma lipoprotein system. FEBS Lett 1973;37:178-182.

2 Blanco-Vaca F, Via DP, Yang CY, et al. Characterization of disulfide-linked heterodimers containing apolipoprotein D in human plasma lipoproteins. J Lipid Res 1992;32:1785-1796.

3 Francone OL, Gurakar A, Fielding C. Distribution and functions of lecithin: cholesterol acyltransclerotic fibromaerase and cholesteryl ester transclerotic fibromaer protein in plasma lipoproteins. Evidence for a functional unit containing these activities together with apolipoproteins A-I and D that catalyzes the esterification and transclerotic fibromaer of cellderived cholesterol. J Biol Chem 1989;264:7066-7072.

4 Drayna D, Fielding C, McLean J, et al. Cloning and expression of human apolipoprotein D cDNA. J Biol Chem 1986;261:16535-16539.

5 Van de Rijn M, Rouse RV. CD34: a review. Appl Immunohistochem 1994;2:71-80.

6 Linn SC, West RB, Pollack JR, et al. Gene expression patterns and gene copy number changes in dermatofibrosarcoma protuberans. Am J Pathol 2003;163: 2383-2395.
7 West RB, Harvell J, Linn SC, et al. Apo D in soft tissue tumors: a novel marker for dermatofibrosarcoma protuberans. Am J Surg Pathol 2004;28:1063-1069.

8 Aiba S, Tabata N, Ishii H, et al. Dermatofibrosarcoma protuberans is a unique fibrohistiocytic tumour expression CD34. Br J Dermatol 1992;127:79-84.

9 Nickoloff BJ. The human progenitor cell antigen (CD34) is localized on endothelial cells, dermal dendritic cells, and perifollicular cells in formalinfixed normal skin, and on proliferating endothelial cells and stromal spindle-shaped cells in Kaposi's sarcoma. Arch Dermatol 1991;127:523-529.

10 Haycox CL, Odland PB, Olbricht SM, et al. Immunohistochemical characterization of dermatofibrosarcoma protuberans with practical applications for diagnosis and treatment. J Am Acad Dermatol 1997;37: 438-444.

11 Cohen PR, Rapini RP, Farhood AI. Expression of the human hematopoietic progenitor cell antigen CD34 in vascular and spindle cell tumors. J Cutan Pathol 1993;20:15-20.

12 Rabinowitz LG, Luchetti ME, Segura AD, et al. Acrally occurring dermatofibrosarcoma protuberans in children and adults. J Dermatol Surg Oncol 1994;20: 655-659.

13 Weiss SW, Goldblum JR. Enzinger and Weiss's Soft Tissue Tumors, 4th edn. Mosby Inc.: St Louis, Missouri, 2001, pp 491-506.

14 Ramani P, Bradley NH, Fletcher CDM. QBEND/10, a new monoclonal antibody to endothelium: assessment of its diagnostic utility in paraffin sections. Histopathology 1990;17:237-242.

15 Weiss SW, Nickoloff BJ. CD34 is expressed by a distinctive cell population in peripheral nerve, nerve sheath tumors, and related lesions. Am J Surg Pathol 1993;17:1039-1045.

16 Hanft VN, Shea CR, McNutt NS, et al. Expression of CD34 in sclerotic ('plywood') fibromas. Am J Dermatopathol 2000;22:17-21.

17 Terrier-Lacombe MJ, Guillou L, Maire G, et al. Dermatofibrosarcoma protuberans, giant cell fibroblastoma, and hybrid lesions in children: clinicopathologic comparative analysis of 28 cases with molecular data-a study from the French Federation of Cancer Centers Sarcoma Group. Am J Surg Pathol 2003;27: 27-39.

18 Jha P, Moosavi C, Fanburg-Smith JC. Giant cell fibroblastoma: an update and addition of 86 new cases from the Armed Forces Institute of Pathology, in honor of Dr Franz M. Enzinger. Ann Diagn Pathol 2007;11: 81-88.

19 Fetsch JF, Laskin WB, Miettinen M. Superficial acral fibromyxoma: a clinicopathologic and immunohistochemical analysis of 37 cases of a distinctive soft tissue tumor with a predilection for the fingers and toes. Hum Pathol 2001;32:704-714.

20 Zelger B, Sidoroff A, Stanzl U, et al. Deep penetrating dermatofibroma versus dermatofibrosarcoma protuberans: a clinicopathologic comparison. Am J Surg Pathol 1994;18:677-686.

21 Goldblum JR, Tuthill RJ. CD34 and factor-XIIIa immunoreactivity in dermatofibrosarcoma protuberans and dermatofibroma. Am J Dermatopathol 1997;19: 147-153.

22 Sachdev R, Sundram U. Expression of CD163 in dermatofibroma, cellular fibrous histiocytoma, and dermatofibrosarcoma protuberans: comparison with 
CD68, CD34, and Factor XIIIa. J Cutan Pathol 2006;33: 353-360.

23 Pozdnyakova O, Mahalingam M. CD99-much ado about nothing. J Cutan Pathol 2007 (in press).

24 Page RN, King R, Mihm Jr MC, et al. Microphalmia transcription factor and NKI/C3 expression in cellular neurothekeoma. Mod Pathol 2004;17:230-234.
25 Fullen DR, Lowe L, Su LD. Antibody to S100a6 protein is a sensitive immunohistochemical marker for neurothekeoma. J Cutan Pathol 2003;30:118-122.

26 Mahalingam M, Alter JN, Bhawan J. Multiple cellular neurothekeomas-a case report and review on the role of immunohistochemistry as a histologic adjunct. J Cutan Pathol 2006;33:51-56. 